JOURNAL DE PHYSIQUE IV

Colloque C4, supplément au Journal de Physique III, Vol. 1, novembre 1991

$\mathrm{C} 4-317$

\title{
STRUCTURE AND PROPERTIES OF NiTi ALLOYS AFTER TREATMENT BY THE POWERFUL ELEC- TRONIC IMPULSES WITH NANOSECOND LENGTH
}

\author{
V. PRIEB \\ An der Fliehburg 19, D-4220 Dinslaken 1, Germany
}

\begin{abstract}
The structural changes, kind of hysteresis loops and shape memory effect in the NiTi-specimens after their irradiation by the impulses electronic beams are investigated. A method for quantitative determination of reversible martensitic deformation under irradiation is proposed. The distribution of internal stresses inside the irradiated zone by means of this method with the use memory is described. Nonequilibrium appearance of structural defects under irradiation is discussed.
\end{abstract}

\section{Introduction}

Investigation of processes which take place in the surface of metallic material during the action of concentrated energy streams is necessary for the further development of the modern impulse technology for a modification of the surface properties by the photonic, ionic or electronic beams /1/. Perspectives of that development are obviously connected with the reaching of nonequilibrium structures under the surface of material after impulse action. Since the number of those nonequilibrium structures is unlimited (it depends on a number of parameters both material and beams) the variety of properties is also unlimited in principle. It is also obvious that the length of the impulse must be shorter than the characteristic time of the reaching of equilibrium of that or another thermodynamic process and the shorter impulse length is the more nonequilibrium state of the system. The nanosecond interval of impulse lengthes, thereby, is a boundary interval because it is near the characteristic time of reaching the total electron-phonon equilibrium.

It is of scientific as well as of practical interest to investigate actions with such length by that the temperature of the electronic subsystem of materials is above that of the phononic temperature, thereby the temperature as a whole system of thermodynamic parameters has no sense. In this case, changes of the structure are possible as a result of the nonequilibrium exchange of the energy between electrons and phonons (i.e. a stimulation of the "soft" phononic modes). Thereby, considerable heating does not take place (there is no considerable increase of the phononic energy). This is even more possible in systems with the peculiarities of the phononic spectrum in the starting state, e.g. $\mathrm{NiTi} / 2 /$.

However, experimental researches of the supershort impulse action on the material surface are very difficult and it is impossible to observe the structural changes in-situ. A lot of information about them has been received for the present as a result of investigations of the final structure and properties of the targets after the impuls had been switched off. Therefore, it is important that a target material should be able to take down the maximum of the information during the impulse length and to preserve ("to memorize") one after it has been switched off. Shape memory alloys are much more perspective for that. They have a high structural sensitivity to different actions (thermal, strength etc.) and a quick response to them. These properties are connected with a thermoelastic martensitic transformation which is responsible for the macroscopic effect of the shape changes (shape memory effect) in a usual situation. 


\section{Experiment}

The equiatomic NiTi-alloy which has all spectrum of the shape memory properties has been choosen as a target material in this work. Starting state of the targets with a thickness of about $1 \mathrm{~mm}$ has been set after hot rolling by means of vacuum annealing at $800^{\circ} \mathrm{C}$ for $0.5 \mathrm{~h}$ which takes off the internal stresses and homogenizes the phase composition $/ 3 /$.

Electronic monoimpulses with the length $\tau_{i}=50 \cdot 10^{-9} \mathrm{~s}$, the current density on maximum (Fig. 1a) $\mathrm{j}_{\mathrm{m}}=10^{3} \mathrm{Acm}^{-2}$ and the initial energy $\mathrm{E}_{0}=250 \mathrm{kev}$ have been used as the concentrate energy streams for the treatment of the target surface. The irradiate scheme is presented in Figure $1 \mathrm{~b}$ where $\mathrm{X}$ is the thickness of the target and $\mathrm{D}$ diameter of the irradiated spot on the target surface. Boundary condition is reached for $D>X>>R_{\text {eff }}$, where $R_{\text {eff }}$ is the projection track of the beam electrons in the material. Investigations of the structure and temperature hysteresis were made by means of X-ray structure diffractometer analysis with a special heating and cooling temperature stage to the goniometer. Special investigations of the structure of deformed specimens the scheme of which is shown in Figure 2 were produced. Structural states of NiTi-specimens were registrated at the different temperatures during the measurements of hysteresis loops and at the etching.

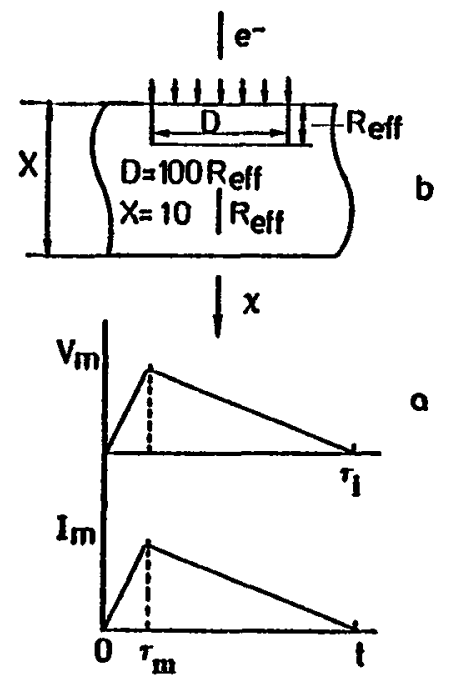

Fig. 1: Oszillograms of current and tension voltage of electronic beam (a) and the irradiate scheme (b)

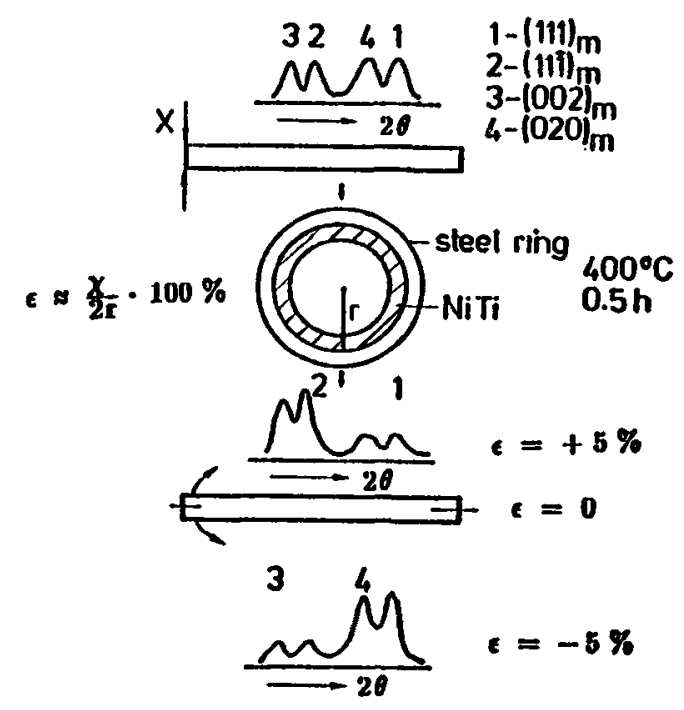

Fig. 2: Scheme of the deformation and diffractogram fragments for started and deformed state of $\mathrm{NiTi}$-specimens

The started state of the specimens at room temperature is completely martensitic. Reflexions $(002) \mathrm{m},(00 \overline{\mathrm{I}})_{\mathrm{m}},(020)_{\mathrm{m}}$, and $(111)_{\mathrm{m}}$ of martensitic B19'-phase with approximately equal intensities are observed on diffractograms. Martensitic temperatures which were determined by means of $\mathrm{X}$-ray hysteresis measurements have next values: $\mathrm{M}_{\mathrm{s}} \div \mathrm{M}_{\mathrm{f}}=(60 \div 40){ }^{\circ} \mathrm{C}$ and $\mathrm{A}_{\mathrm{s}} \div \mathrm{A}_{\mathrm{f}}=(80 \div$ 100) ${ }^{\circ} \mathrm{C}$. It corresponds to temperature ranges which were determined by means of acoustic emission measurements /4/. As a result of the irradiation, high-temperature B2-phase arises, and diffractograms outline two-phase $\mathrm{B} 2+\mathrm{B} 19^{\prime}$-state of the NiTi-specimens at room temperature (Fig. 3). This means that the $\mathrm{M}_{\mathrm{S}}$-temperature is changed. Moreover, diffractograms outline a change of the intensities of the martensitic reflexes $(11 \overline{1})_{\mathrm{m}}$ and $(111)_{\mathrm{m}}$, whereas the intensities of the reflexes $(002)_{\mathrm{m}}$ and $(020)_{\mathrm{m}}$ remain equal to each other.

Curves of the temperature hysteresis for irradiated specimens indicate that the martensitic temperatures have really changed (Fig. 3). Consequently, the shape of the hysteresis curves has 


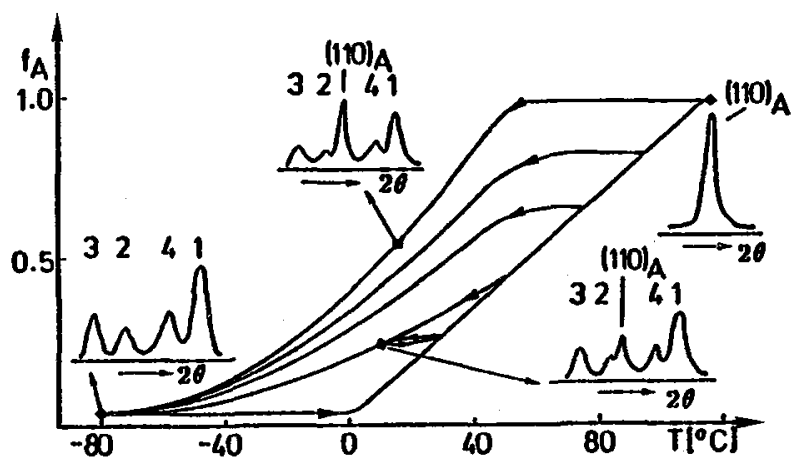

Fig. 3: Loops of temperature hysteresis for the completed and uncompleted transformation cycles and diffractogram fragments for irradiated NiTi-specimens

changed from the form which is characteristic for the NiTi $\left(A_{s}>M_{s}\right)$ to the one characteristic for $\mathrm{Cu}$-based shape memory alloys $\left(\mathrm{A}_{\mathrm{s}}<\mathrm{M}_{\mathrm{s}}\right)$. The thermocycling at temperature hysteresis measurements had begun by the cooling from the room temperature. In the irradiated zone of all specimens the phase composition corresponds to a certain incompleteness of the reverse transformation (Fig. 3). Etching of the irradiated specimens to a depth of about $200 \mu \mathrm{m}$ leads to the restoration of the start hysteresis loops. Corresponding changes of the phase composition do also occur. The intensity of the austeniti reflexion (110) sharply decreases.

By etching the intensity ratio of the martensitic reflexes $(11 \overline{1})_{\mathrm{m}}$ and $(111)_{\mathrm{m}}$ changes. To the depth $\mathrm{x}=30 \mu \mathrm{m}$ it becomes equal to the unit then it reverses and at the depth of about $60 \mu \mathrm{m}$ deviation from the unit it reaches its maximum again. To the depth $120 \mu \mathrm{m}$ this ratio returns to its original state.

To compare the irradiation influence on the martensitic phase with the strain action, diffractograms from the compressed and the tensile side of the flat $\mathrm{NiTi}$-specimens after annealing in the steel rings and deformation by means of the straightening were used (Fig. 2). Not only the ratio of the intensities of martensitic reflexions $(11 \overline{1})_{\mathrm{m}}$ and $(111)_{\mathrm{m}}$ but also the $(020)_{\mathrm{m}}$ and $(002)_{\mathrm{m}}$ changes by similar means on the diffractograms of the deformed specimens as distinct from the irradiated specimens.

The shape memory effect increases in comparison to the starting value. However, the irreversible strain which remains after heating up to above $\mathrm{A}_{\mathrm{f}}$ and unloading also increases. Temperature hysteresis loops which are defined by means of measurements of the shape memory effect correspond to those measured by $\mathrm{X}$-ray method. Sharper changes of the reversible strain in the beginning of the direct and in the end reversible transformation are - unlike the curves from Figure 3 - can be connected with layers of the material which lies below the irradiated zone and has the properties of the starting state and takes part in the general deformation.

\section{Discussion}

The deviation of the ratio $\mathrm{K}_{12}$ of the martensitic reflexion $(111)_{\mathrm{m}}$ intensity referred to as $\mathrm{I}_{1}$, to the reflexion $(11 \overline{1})_{m}$ intensity as $\mathrm{I}_{2}$, as the unit is connected with the monodomainization process of martensitic crystals by the action external or internal shear stress, as it follows from the results which were received for the deformed specimens (Fig. 2) and as it directly follows from the nature of the shape memory effect itself (Figs. 2a-c/5/). Favourable to these stresses orientation variants of the martensite grow on account of the unfavourable one, e.g. by means of the retwinning if the martensitic substructure which is a result of the lattice invariant deformation during the direct 
martensitic transformation is twinned as in $\mathrm{NiTi}$. The shear value $\gamma$ can simply be connected with the transformation (clear) shear $\alpha$ :

$$
\gamma=\alpha\left(1-\mathrm{n}^{-} / \mathrm{n}^{+}\right) /\left(1+\mathrm{n}^{-} / \mathrm{n}^{+}\right)
$$

Where $\mathbf{n}^{+}$is the number of crystal planes with a "right" shear (e.g., towards the martensitic shear) and $n^{-}$with a "left" shear (in the opposite direction). The ratio $n^{+} / n^{-}$can lead to the intensity ratio $\mathrm{I}_{1} / \mathrm{I}_{2}$ for planes $(111)_{\mathrm{m}}$ and $(11 \overline{1})_{\mathrm{m}}$, therefore:

$$
\epsilon=\epsilon_{\max }\left(1-\mathrm{K}_{12}\right) /\left(1+\mathrm{K}_{12}\right) \delta_{12}
$$

where $\delta_{12}$ is a rearranged symbol. Parameter $\mathrm{K}_{12}$ changes from 0 to 1 according to sense of equ. (2). As this condition is always implemented it is necessary to rearrange the numerator and denominator in $\mathrm{K}_{12}$, i.e. they must be exchanged by their indexes in equ. (2). Symbol $\delta_{12}$ serves this aim. The starting indices had been empirically chosen so that " + " corresponds to the compressed side and "-" to the tensile side of deformed specimens. The examination of this semiempirical equ. (2) for the beforehand deformed specimens (Fig. 2) showed the good agreement between the given and calculated - by means of equ. (2) $\left(\epsilon_{\max }=10 \%\right.$ for the NiTi-alloys $)$ - value of the strain. However, this agreement only takes place in strain values up to about $5 \%$. If the strain is larger than $5 \%$ this agreement gets inaccurate. This means not only monodomainization of the martensitic crystals as is supposed in equ. (2) but also their rotation as a whole takes place. It is connected with a plastic strain which takes place in the case of large deformation and deteriorates the shape memory effect.

The comparison of diffractograms of irradiated and deformed specimens together with the facts explained above allow the conclusion that the electronic impulse irradiation exerts the orientating effect on the martensitic phase. Perhaps this effect is not identical with the action of external stress (the behaviour of the intensity ratio of the martensitic reflexes $(020)_{m}$ and $(002)_{m}$ is different) but one is similar. The difference may be connected with the peculiar deformation geometry, e. $g$. "hydrostatic" two-dimensional tension/compression acts in atomic planes which the axis $\mathrm{x}$ of the beam is perpendicular to (Fig. 1). This geometry can be assumed from the symmetry of action. The heating up above the finish temperature of the reversible transformation $A_{f}$ (Fig. 3) for a short time does not change the state of the irradiated specimens, whereas the annealing at the temperature $400^{\circ} \mathrm{C}$ for 0.5 hours bisect the ratio $\mathrm{K}_{12}$ in equ. (2). It means that the orientation of the martensitic crystals is not only immediately influenced by the electronic beam but also by means of the induction of internal stresses. Nonequilibrium defects of the structure which are induced by the irradiation can in this case be the cause of such stresses in the action zone.

The distribution of the martensitic strain across the depth of the irradiated zone was calculated with the help of equ. (2) and experimental data which were received from step-to-step-etching, as shown above (Fig. 4). This distribution is not only a representation of the distribution of internal orientating stresses with irreversible character but also, almost naturally, the distribution of dynamic shear stresses which were acting during the impulse length in the irradiated zone and, as a result of that, the NiTi-alloy provides us with the possibility to take a picture of this distribution which is qualitatively represented in Figure 5. Figures 4 and 5 outline that the internal stresses are in a zone of depth of $120 \mu \mathrm{m}$ and have the different sign, tensile stresses under the surface and compressed in a depth from 30 to $120 \mu \mathrm{m}$. Depth $\chi=120 \mu \mathrm{m}$, where the stresses decrease to zero correspond to the projection track $\mathrm{R}_{\text {eff }}$ of the beam electrons with the starting energy $\mathrm{E}_{0}$ in the alloy with the density $\rho$ which is calculated from the empirical Widdington-Tomson's formula [6]:

$$
\mathrm{R}_{\text {eff }} \stackrel{N}{=} \mathrm{E}_{0}^{2} / \rho \mathrm{b} \cong 130 \mu \mathrm{m}
$$

with $\mathrm{b}$ an empirical parameter, connected with the atom number of the material. It means that the structural changes take only place in the zone of immediate energy losses of the beam electrons whose distribution $Q(\chi)$ is shown in Figure 5 as a curve of cupola-shape with a maximum in the 


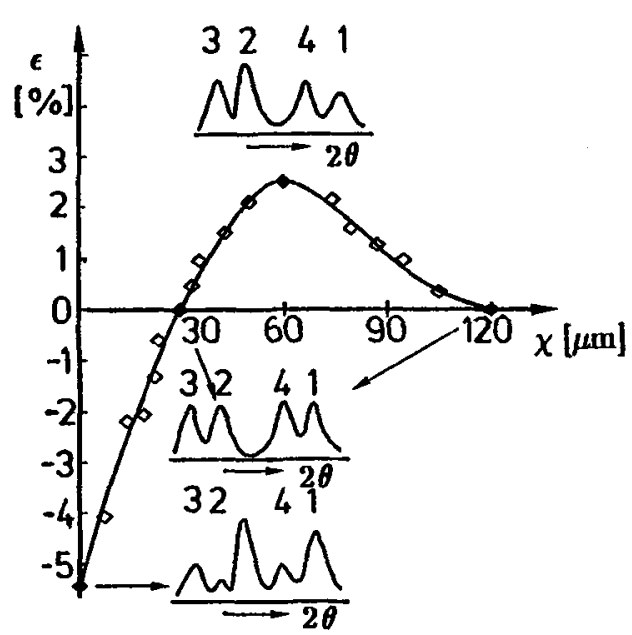

Fig. 4: Diffractogram fragments and unelastic strain distribution in irradiated $\mathrm{NiTi}$

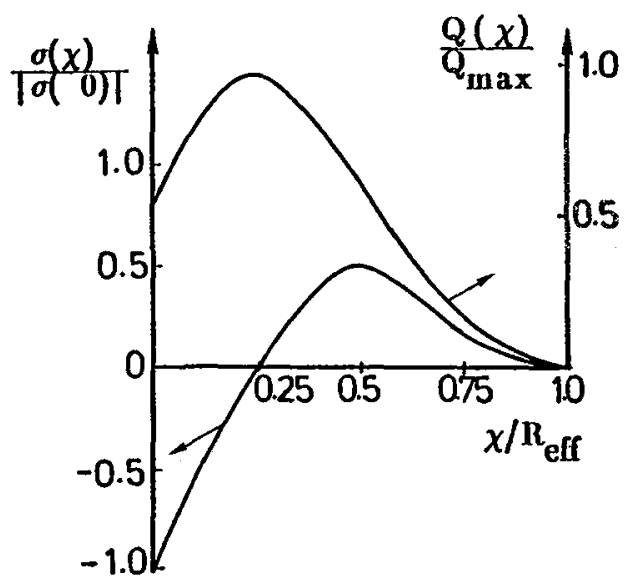

Fig. 5: Distribution of dynamic stresses and electron energy lossed in alloys at irradiation

depth $\mathrm{x} \cong 0.25 \mathrm{R}_{\text {eff }}$. This depth is about $30 \mu \mathrm{m}$ (equ. 3) and the stresses change their sign here (Figs. 4,5).

It can be seen in Figure 5 that the curve of the stress distribution can be qualitatively received by means of direct differentiation of the distribution $\mathrm{Q}(\chi)$. For the one-dimensional case differentiation along the axes $x$ of rotation symmetry is received:

$$
\sigma(\chi)=-\operatorname{grad}_{\mathrm{X}}[\mathrm{A} \cdot \mathrm{Q}(\chi)]
$$

where $A$ is the parameter whose determination allows a qualitative correspondence between the distribution of the stresses and the energy losses to fix.

The results of the measurement hysteresis curves at the uncompleted transformation cycles (Fig. 3 ) and the structural state of irradiated specimens at room temperature show that the temperature in the irradiated zone during the impulse length was not higher than the finish temperature $A_{f}$. The heating temperature can be more exactly determined from Figure 3 as $T_{h} \approx 60^{\circ} \mathrm{C}$. The calculated temperature based on an adiabatic approximation using the maximum values $\mathrm{j}_{\mathrm{m}}$ and $\mathrm{U}_{\mathrm{m}}$ (Fig. 1a) is:

$$
\mathrm{T}_{\max }=\mathrm{j}_{\mathrm{m}} \cdot \mathrm{U}_{\mathrm{m}} \cdot \tau_{\mathrm{i}} / \mathrm{c}_{\mathrm{p}} \cdot \rho \cdot \mathrm{R}_{\mathrm{eff}}
$$

where $c_{p}$ is the specific heat capacity at the constant pressure. At the depth of the irradiated zone $\mathrm{R}_{\text {eff }}$ about $100 \mu \mathrm{m}$ (Fig. 4), $\mathrm{T}_{\max } \cong 600^{\circ} \mathrm{C}$. Thus the value $\mathrm{T}_{\mathrm{h}} \cong 60^{\circ} \mathrm{C}$ is real.

In spite of such an insignificant value of the heating temperature, the temperature gradient in action zone with the depth about $100 \mu \mathrm{m}$ can be enough for the creation of significant thermostresses whose distribution in shown in Figure 5. This distribution is at any rate similar by appearance to the calculating on the basis of a thermoelastic model. However, the stresses which are received in this model are not shear, but hydrostactic (by boundary condition $D>>R_{\text {eff }}$ ) since the thermal expansion coefficient is taken in calculations as a scalar value for isotropic material.

On the other hand an athermal nature of the appearance of non-equilibrium structural defects in metallic material under irradiation by the powerful impulses with nanosecond length can be supposed which corresponds to the physical sense of equations (3) and (4). It can be connected with a nonequilibrium energy exchange between the excited electronic subsystem and the "cold" 
crystal lattice. Such an example is given by the excitonic mechanism of the fracture of insulators at a similar action. Thus the plasmons can effect such cooperative excitations in metallic material. In any case, additional detailed investigations are necessary for a further discussion.

The broadening of the temperature intervals of the direct and reverse martensitic transformation in this work, which is connected with the structural inhomogenity through the section of the specimen after its treatment by electronic impulses is higher than it was received by means of creation $\mathrm{NiTi}$-diffusion layers with changed composition $/ 7 /$.

The increase of values of reversible and irreversible strain does not occur on principle, but investigation of such an influence of irradiation on the shape memory effect is of practical interest since it is connected with the problem of the stability of this effect at the irradiation. This problem occurs in practical applications of shape memory alloys in space or in nuclear/thermonuclear reactors $/ 8 /$. Instability of the memory properties can be connected with the migration of the structural defects which were induced by irradiation under the action of external stresses, as well as by the change of martensitic temperatures as it was shown above.

It can be supposed that the final state of alloys after irradiation by the electronic impulses with nanosecond length results from the changes of the exchange interaction potential:

$$
\mathrm{U}(\chi)=\mathrm{A} \cdot \mathrm{Q}(\chi)
$$

in the superaroused state with the "cold" iones and "hot" electrons. Equation 6 follows from physical sense equation 4. All spectrum of the metastable states which is characteristic for this alloy as well as considerable dynamic stresses can be reached as a result of such changes of the potential.

\section{Conclusion}

The use of NiTi-shape memory alloys as a target is productive for the investigations of the powerful electronic impulse action on the metallic surface as was shown in the results of this work. The methods described in this paper allow with the help of NiTi-targets to achieve the following characteristics of such treatment:

1. The heating temperature in irradiated zone.

2. The depth of irradiated zone.

3. The stresses distribution in irradiated zone.

Moreover, such treatment is important for the use of shape memory alloys in practice; i.e. it allows

- the temperature hysteresis loops to modify considerably,

- the problem of the use of the shape memory alloys in the irradiation conditions to investigate and, in that way, to decide.

It presupposes, however, further investigations in this direction.

\section{Acknowledgement}

The irradiation of specimens was carried out in electronic impuls guns [1] of research centres of USSR.

\section{References}

11/ Bound papers from Symposium on Powerful Current Electronic USSR 8 (1990)

/2/ PERKINS, J., Met. Trans. 4 (1973) 2475

/3/ PRIEB, V.E., PASKAL, YU.I., MONASEVICH, L.A. et al., MiTOM, Moscow 9 (1979) 62

/4) PRIEB, V.E., LUBIVOY, V.P., Metallophysic, Kiev 10 (1988) 179

15/ HORNBOGEN, E., Pract. Met. 26 (1989) 279

/6/ PRIEB, V.E., PINKIN, V.F., LYS, V.F., in [1], p. 184

17) SAVADA KADSUO, HAYASI KADZUCHIKO, Announce on invention N57-232082, Japan, publ. 05.04.1984, IKI c22c 1/00, B23K 20/00

18/ NISHIKAVA NASACHIRO, VATANABBA KENJI, J. Atom. Energy Soc. Jap. 1 (1987) 2 